\title{
The Extended Touschek Lifetime
}

\author{
Hana M. Bizek
}

Argonne National Laboratory, 9700 South Cass Ave, Argonne, IL. 60439

\section{Section 1: Introduction.}

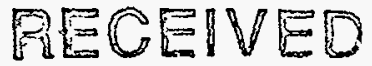

FEB $0819=0$

\section{OSTI}

With the advent of synchrotron radiation sources, the issue of beam lifetime becomes increasingly important. Users of these machines need to perform experiments which seldom last 15 minutes, but require hours for their completion. Therefore, the beam should circulate stably for hours. The beam of the Advanced Photon Source (APS) storage ring at Argonne National Laboratory is assumed to circulate stably for a minimum of 10 hours.

The main contributions to the total beam lifetime (which is the inverse of the loss rate) come from residual gas scattering and Touschek scattering. The residual gas scattering is comprised of single Coulomb scattering and bremsstrahlung. The single-Coulomb scattering involves elastic collisions, while bremsstrahlung involves inelastic collisions, between the bunch and the surrounding residual gas. In our calculation we take the gas to be nitrogen (atomic number $Z=7$ ) at a pressure of 1 nTorr. Touschek scattering involves scattering of particles within the bunch, transferring energy among themselves. Such an energy transfer, if large enough, may eject the particle out of the bunch, thus causing it to be lost.

When introducing dispersion into the beam lifetime, we direct our attention to Touschek scattering. A particle passing through the dispersive region will induce a horizontal betatron oscillation which, when passing through skew components, will be coupled into vertical motion. The amount of coupling is expressed in terms of the coupling coefficient, $\chi$. If the coupling coefficient is large enough, the resulting vertical oscillations may exceed the normally small vertical admittance of the ring. Thus the particles may be lost before the energy loss exceeds the acceptance of the rf bucket. The inclusion of this loss mechanism is called the extended Touschek lifetime. The end result is to place an upper limit on the coupling coefficient, beyond which the lifetime becomes smaller than the aforementioned minimum lifetime for stable storage ring operation.

Let us not forget the residual-gas lifetime. As pointed out, the calculation of this lifetime is done for the very low pressure of 1 nTorr. If the pressure is higher, the residual-gas lifetime will be smaller. This will further reduce the total beam lifetime, causing it to slip deeper below the minimum lifetime for stable storage ring operation.

We begin this article by reviewing the Touschek integral and the associated limits of integration. The program ZAP [1] has been altered to take into account the possible loss due to induced betatron oscillations. At each lattice position the energy loss required to produce, by coupling, a vertical oscillation that exceeds the vertical aperture is calculated. When this energy loss is less than the rf bucket half-height, it replaces the rf bucket halfheight in the Touschek integral.

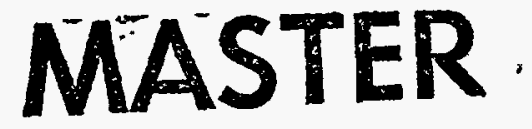

DSTARUTION OF THIS DOCUNEENT IS UNLLGTED a 


\section{Section 2: $\quad$ The Touschek integral and its limits.}

The normal Touschek integral has been derived in Bruck [2]. Starting with the Moller differential cross section, one does the appropriate integrals. The calculation is messy, but straightforward. Taking the mean of the product of velocity and cross section, one obtains

$$
\overline{v \sigma}=2 \pi r_{0}^{2} c \int_{q \text { lower }}^{q u=\frac{\Delta p_{r f}}{r}} \frac{1}{q^{4}}\left(\left(\frac{\gamma q}{\Delta p_{r f}}\right)^{2}-\frac{1}{2} \ln \left(\frac{\gamma q}{\Delta p_{r f}}\right)^{2}-1\right) \frac{\exp \left(-\frac{q^{2}}{\delta p_{x}^{2}}\right)}{\sqrt{\pi} \delta p_{x}} q d q
$$

where $r_{0}=2.82 \times 10^{-15} \mathrm{~m}$ is the classical electron/positron radius, $c$ is the speed of light, $v$ is the relative velocity of two electrons or positrons, $q$ is the velocity, divided by $c$, of each electron or positron in the center-of-mass of the pair, and $\gamma=1 / \sqrt{1-v^{2} / c^{2}}$ is the relativistic factor. $\delta p_{x}$ is the standard deviation of the Gaussian distribution of the radial components of the momentum $p_{x}$. The lower limit corresponds to the of bucket half-height, because $\Delta p_{r f} / p_{0}=\Delta p_{r f} / \gamma$, since the momentum of the synchronous particle $p_{0}$ is written in units of $m_{0} c$. For relativistic particles we have $p_{0}=m_{0} \gamma v / m_{0} c \approx \gamma$.

We introduce the following change of variables

$$
u=\left(\frac{q}{\delta p_{x}}\right)^{2} \quad \epsilon_{r f}=\left(\frac{\Delta p_{r f}}{\gamma \delta p_{x}}\right)^{2} \quad \frac{u}{\epsilon_{r f}}=\left(\frac{\gamma q}{\Delta p_{r f}}\right)^{2} \quad \frac{1}{u^{2}}=\left(\frac{\delta p_{x}}{q}\right)^{4} \quad d u=\frac{2 q d q}{\left(\delta p_{x}\right)^{2}} .
$$

With this variable change, the limits of integration are

$$
u_{\text {lower }}=\left(\frac{q_{\text {lower }}}{\delta p_{x}}\right)^{2}=\left(\frac{\Delta p_{r f}}{\gamma \delta p_{x}}\right)^{2}=\epsilon_{r f} \quad \text { and } \quad u_{\text {upper }}=\left(\frac{q_{u p p e r}}{\delta p_{x}}\right)^{2}=\infty .
$$

Inserting Eqs. (2) and (3) into Eq. (1) we obtain

$$
\overline{v \sigma}=\frac{\sqrt{\pi} r_{0}^{2} c}{\delta p_{x}^{3}} \int_{\epsilon_{r f}}^{\infty} \frac{1}{u^{2}}\left(\frac{u}{\epsilon_{r f}}-\frac{1}{2} \ln \left(\frac{u}{\epsilon_{r f}}\right)-1\right) \exp (-u) d u .
$$

The normal Touschek lifetime formula is

$$
\frac{1}{\tau_{n} T}=\frac{N}{\gamma^{2} V} \overline{v \sigma}=\frac{\sqrt{\pi} r_{0}^{2} c N}{\gamma^{2}\left(\delta p_{x}\right)^{3} V} \int_{\epsilon_{r f}}^{\infty} \frac{1}{u^{2}}\left(\frac{u}{\epsilon_{r f}}-\frac{1}{2} \ln \left(\frac{u}{\epsilon_{r f}}\right)-1\right) \exp (-u) d u
$$

with $\epsilon_{r f}$ given in Eq. (3). Here $N$ is the number of positrons per bunch, $V$ is the bunch volume, and $\tau_{n T}$ is the normal Touschek lifetime.

Normal Touschek lifetime increases as the coupling coefficient gets larger. However, if one includes horizontal oscillations, which occur in the dispersive region, an increased coupling coefficient can lead to vertical oscillation, which may exceed the normally small vertical apertures. When one includes this effect, one sees that increased coupling leads to decreased lifetime. Our results indeed bear this out.

Next we suppose a bunch travels in the dispersive region of the storage ring and loses energy $\Delta p_{1} / p_{0}$. This produces a horizontal betatron displacement and its derivative, which are given in terms of the dispersion function $\eta$ and its derivative $\eta^{\prime}$ as

$$
x=\eta\left(\frac{\Delta p_{1}}{p_{0}}\right) \quad \text { and } \quad x^{\prime}=\eta^{\prime}\left(\frac{\Delta p_{1}}{p_{0}}\right) .
$$


This displacement and slope leads to the Courant-Snyder invariant

$$
W_{x}=\left(\frac{\Delta p_{1}}{p_{0}}\right)^{2} \frac{1}{\beta_{x}}\left(\eta^{2}+\left(\beta_{x} \eta^{\prime}-\frac{1}{2} \beta_{x}^{\prime} \eta\right)^{2}\right)=\left(\frac{\Delta p_{1}}{p_{0}}\right)^{2} \mathcal{H}
$$

where $\mathcal{H}$ is the so-called Sands factor [3]. The function $\mathcal{H}$ is shown in Fig. 1 for the APS half-sector of total length $L=13.8 \mathrm{~m}$. Then the coupling coefficient $\chi$ is the ratio of the Courant-Snyder invariants $W_{y} / W_{x}$. The maximum $W_{y}$ is determined by the vertical admittance, $A_{y}$

$$
W_{y ; \max }=A_{y}=\frac{a^{2}}{\beta_{y}}
$$

where $a$ is the vertical half-aperture at the center of the insertion region and $\beta_{y}$ is the vertical beta function at the center of the insertion region. For the present study, $\beta_{y}=10 \mathrm{~m}$, the value at insertion of the APS storage ring, and $a$ is assumed to be $2 \mathrm{~mm}, 3 \mathrm{~mm}$, and $4 \mathrm{~mm}$. Combining Eqs. (7) and (8) we obtain

$$
\begin{gathered}
\left(\frac{\Delta p_{1}}{p_{0}}\right)=\left(\frac{\Delta p_{1}}{\gamma}\right)=\sqrt{\frac{a^{2}}{\chi \beta_{y} \mathcal{H}}} . \\
\epsilon_{1}=\left(\frac{\Delta p_{1}}{\gamma \delta p_{x}}\right)^{2} .
\end{gathered}
$$

For the extended Touschek lifetime, Eq. (5) is replaced by

$$
\frac{1}{\tau_{e T}}=\frac{N}{\gamma^{2} V} \overline{v \sigma}=\frac{\sqrt{\pi} r_{0}^{2} c N}{\gamma^{2}\left(\delta p_{x}\right)^{3} V} \int_{\epsilon_{0}}^{\infty} \frac{1}{u^{2}}\left(\frac{u}{\epsilon_{1}}-\frac{1}{2} \ln \left(\frac{u}{\epsilon_{1}}\right)-1\right) \exp (-u) d u .
$$

In this equation $\epsilon_{0}$ is the smaller of either $\epsilon_{1}$ or $\epsilon_{r f}$. The lower limit in Eq. (11) depends on the Twiss parameters, dispersion and its derivative (through $\mathcal{H}$ ), the coupling coefficient $\chi$, and the vertical half-aperture $a$. It is the task of this article to examine the extended Touschek lifetime and to calculate its influence on the total beam lifetime.

\section{Section 3: The method of calculation.}

The extended Touschek integral in Eq. (11) together with the lower limit in Eq. (10) are evaluated using the code ZAP, which is modified for this purpose. ZAP has the option of calculating the normal Touschek lifetime or the extended Touschek lifetime. If the latter option is used, the code asks the user to input the value of the vertical half-aperture $a$ in $\mathrm{mm}$, followed by the value of $\beta_{y}$, the vertical beta function at the center of the insertion region in $\mathrm{m}$.

The main difference between the routine on the extended Touschek lifetime and the routine on the normal Touschek lifetime is that the former also treats betatron oscillations produced in the dispersive region. The dispersive region is typified by the non-zero Sands factor $\mathcal{H}$. If $\mathcal{H}=0$, the lower limit will be $\epsilon_{r f}$ and Eq. (11) will be identical with Eq. (5), the normal Touschek lifetime. 
On the other hand, if $\mathcal{H} \neq 0$, then ZAP calculates the lower limit due to betatron oscillations produced in the dispersive region via Eqs. (9) and (10). If $\epsilon_{1}<\epsilon_{r f}$ then $\epsilon_{1}$ is used in Eq. (11) and the loss mechanism of extended Touschek lifetime calculation is used. If $\epsilon_{1} \geq \epsilon_{r f}$ then $\epsilon_{r f}$ is used in Eq. (11) and the loss mechanism of normal Touschek lifetime calculation is used. Having decided what the lower limit is going to be, ZAP proceeds to evaluate the Touschek integral of Eq. (11), or its special case, Eq. (5). The average of lifetimes at individual lattice points is taken.

ZAP accepts horizontal and vertical emittances, rather than the coupling coefficient. So we must obtain those numbers. To calculate the horizontal and vertical emittances, one uses the well-known formulas

$$
\epsilon_{x}=\frac{\epsilon_{n}}{1+\chi} \quad \text { and } \quad \epsilon_{y}=\frac{\chi \epsilon_{n}}{1+\chi}
$$

where $\epsilon_{n}=8.2 \times 10^{-9} \mathrm{~m}$-rad is the natural emittance and $\chi$ is the coupling coefficient.

The energy loss is given in the list of parameters below. From this one obtains the synchrotron frequency and, given the rms bunch length ${ }^{1}$, the rms momentum spread $\sigma_{p} / p$ is related to the rms bunch length $\sigma_{\ell}$ via the formula

$$
\frac{\sigma_{p}}{p}=\frac{\sigma_{\ell} \omega_{s}}{\alpha c}
$$

where $\omega_{s}=2 \pi f_{s}$ is the synchrotron angular frequency and $\alpha$ is the momentum compaction.

Some other parameters [4] relating to the APS storage ring are:

Beam energy

Number of positrons per bunch

Natural rms momentum spread

Minimum rms bunch length, dipoles

Minimum rms bunch length, including insertion devices

Synchrotron radiation energy loss, dipoles

Synchrotron radiation energy loss, including insertion devices

Momentum compaction

$\mathrm{RF}$ frequency

RF voltage
7

$1.2 \times 10^{11}$

$0.96 \times 10^{-3}$

0.53

0.58

5.45

6.90

$2.28 \times 10^{-4}$

351.93

9.5
$\mathrm{GeV}$

$\mathrm{cm}$

$\mathrm{cm}$

$\mathrm{MeV} /$ turn

$\mathrm{MeV} /$ turn

$\grave{\mathrm{MHz}}$

MV

The given number of positrons/bunch corresponds to $5.22 \mathrm{~mA}$ current. This current is fixed throughout the whole calculation.

\section{Section 4: Presentation and analysis of results.}

The extended Touschek lifetime as a function of the coupling coefficient is shown graphically in Figs. 2 through 5. Figures 2 and 3 show the extended Touschek lifetime calculation for an $\mathrm{rf}$ bucket half-height of $2.8 \%$ and rms bunch lengths of $2 \mathrm{~cm}$ and $1 \mathrm{~cm}$, respectively.

\footnotetext{
${ }^{1}$ The rms bunch length is assumed to be $1 \mathrm{~cm}$ and $2 \mathrm{~cm}$.
} 
Figures 4 and 5 show the extended Touschek lifetime calculation for an rf bucket half-height of $2 \%$ and rms bunch lengths of $2 \mathrm{~cm}$ and $1 \mathrm{~cm}$, respectively. The normal Touschek lifetime is included for comparison.

The numbers in the legends of the figures refer to the vertical half-aperture $a$ and are used to calculate the lower limit of the extended Touschek lifetime integral in Eq. (9). The normal Touschek lifetime (marked as "normal Touschek" in the figures' legends) is calculated for each coupling coefficient by using the appropriate energy loss corresponding to the appropriate bucket half-height, as spelled out in the parameters at the end of Section 3.

Obviously, both the extended and the normal Touschek lifetimes are greatest for 2-cm rms bunch length and for $0.028 \mathrm{rf}$ bucket half-height, as seen in Fig. 2. On the other hand, these lifetimes are smallest for $0.02 \mathrm{rf}$ bucket half-height and an $\mathrm{rms}$ bunch length of $1 \mathrm{~cm}$, as seen in Fig. 5.

Of more practical interest is the total beam lifetime. In general, the total beam lifetime is the inverse of the sum of the inverses of residual gas and Touschek lifetimes. If the limits due to oscillations in the dispersive region are included, the Touschek lifetime is replaced by extended Touschek lifetime, as calculated and displayed in Figs. 2-5. We thus have

$$
\begin{array}{ll}
\frac{1}{\tau_{t o t-n T}}=\frac{1}{\tau_{\text {gas }}}+\frac{1}{\tau_{n T}} & \text { total with normal Touschek } \\
\frac{1}{\tau_{\text {tot-eT }}}=\frac{1}{\tau_{\text {gas }}}+\frac{1}{\tau_{e T}} & \text { total with extended Touschek }
\end{array}
$$

where $\tau_{g a s}$ is the residual gas lifetime, $\tau_{n T}$ is the normal Touschek lifetime, $\tau_{e T}$ is the extended Touschek lifetime, $\tau_{t o t-n T}$ is the total beam lifetime with normal Touschek lifetime and $\tau_{t o t-c T}$ is the total beam lifetime with extended Touschek lifetime. The total beam lifetime is displayed in Figs. 6 - 9 for vertical half-apertures of $2 \mathrm{~mm}, 3 \mathrm{~mm}$, and $4 \mathrm{~mm}$, as shown in the legends to those figures. Also, in the legends "extended" stands for $\tau_{t o t-e T}$, calculated via Eq. (15), and "normal" stands for $\tau_{t o t-n T}$, calculated via Eq. (14).

For a given coupling coefficient, $\tau_{\text {tot-eT }}$ is always smaller than $\tau_{\text {tot-nT }}$. For a fixed coupling coefficient $\chi$, the difference between $\tau_{\text {tot }-n T}$ and $\tau_{\text {tot-eT }}$ is called the discrepancy, which quantifies the influence of oscillations in the dispersive region. The discrepancy increases with increasing coupling and decreasing vertical half-aperture, for fixed beam current, fixed rms bunch length, and fixed rf bucket half-height. The discrepancy also increases with decreasing. rms bunch length if all other parameters are kept fixed. This is seen from Figs. 6 and 7 on one hand, and Figs. 8 and 9 on the other.

From the graphs of the total beam lifetime it can be seen that the largest discrepancy occurs for higher bucket half-height, lower rms bunch length, lower vertical half-aperture, and higher coupling. Such a case occurs in Fig. 7; for $2.8 \% \mathrm{rf}$ bucket half-height, 1-cm rms bunch length, 2-mm vertical half-aperture, and $100 \%$ coupling, the discrepancy between $\tau_{\text {tot-nT }}$ and $\tau_{\text {tot }-\mathrm{cT} T}$ is about 6 hours. This is a large enough number to be observed, particularly since the total lifetime with normal Touschek scattering is about 18 hours in this case.

This is a somewhat extreme case, since it appears unlikely that we will use such a high coupling. For the case of Fig. 7, however, even smaller coupling coefficients experience noticeable discrepancy. For $30 \%$ coupling coefficient, the discrepancy turns out to be about 2 hours. This corresponds to the total lifetime with normal Touschek scattering of about 17.86 hours. We should be able to see that. 
As we stated at the beginning of this article, the influence of betatron oscillations in the dispersive region may cause the total lifetime to slip below the prescribed minimum lifetime for optimum storage ring operation, or come perilously close to doing so. This can happen even for pressures as low as $1 \mathrm{nTorr}$ if the rf bucket half-height, the rms bunch length, and the vertical half-aperture are small enough and the coupling coefficient is large enough. In Fig. 9, we have such a case. For $2-\mathrm{mm}$ vertical half-aperture and $100 \%$ coupling, $\tau_{\text {tot-eT }}$ is 10.9 hours, while $\tau_{\text {tot-nT }}$ is 15.52 hours. (Recall that the minimum lifetime for optimum operation of the APS storage ring is 10 hours.) For a pressure of just $1.2 \mathrm{nTorr}$, the residualgas lifetime is about 15.66 hours and the extended Touschek lifetime is about 26 hours, which means that the total beam lifetime is, from Eq. (15), 9.51 hours. The inescapable conclusion is: under certain conditions, inclusion of betatron oscillations in the dispersive region may cause the total beam lifetime to become quite small, even at low pressures. With higher pressures the situation can only get worse, because the residual-gas lifetime will be correspondingly lower. In such a case, not only bunches having very high coupling, but even bunches of lower coupling may be affected.

On the other side of the scale, the lowest discrepancy occurs for lower bucket half-height, higher rms bunch length, higher vertical half-aperture, and lower coupling. Such a case occurs in Fig. 8 ; for $2 \%$ rf bucket half-height, 2 -cm rms bunch length, 4-mm vertical halfaperture, and $40 \%$ coupling, the discrepancy between $\tau_{\text {tot }-n T}$ and $\tau_{\text {tot }-\mathrm{eT}}$ is about 0.006 hours. Given the fact that the calculated total lifetime with normal Touschek scattering is about 17 hours, the discrepancy is truly negligible.

\section{Section 5: Conclusion and acknowledgments.}

Inclusion of betatron oscillations in the dispersive region when calculating the total beam lifetime is not generally considered in the theory. It should be. As we have seen, consideration of such oscillations becomes of some importance for lower vertical half-apertures, higher coupling coefficients, higher rf bucket half-heights and lower rms bunch lengths.

The author wishes to thank Dr. Glenn A. Decker for suggesting this problem and Dr. Edwin A. Crosbie for assistance and valuable discussions.

\section{References}

[1] Zisman, M. S., Chattopadhyay, S., Bisognano, J. J., ZAP User's Manual, LBL-21270 UC-28, December 1986.

[2] Bruck, H., Circular Particle Accelerators, Los Alamos National Laboratory, LA-TR-7210, Rev.

[3] Sands, Matthew, The Physics of Electron Storage Rings, SLAC 121, May 1979.

[4] Crosbie, Edwin A., Revised Lattice for the APS Storage Ring, LS-142, August 1989. 
Sands factor

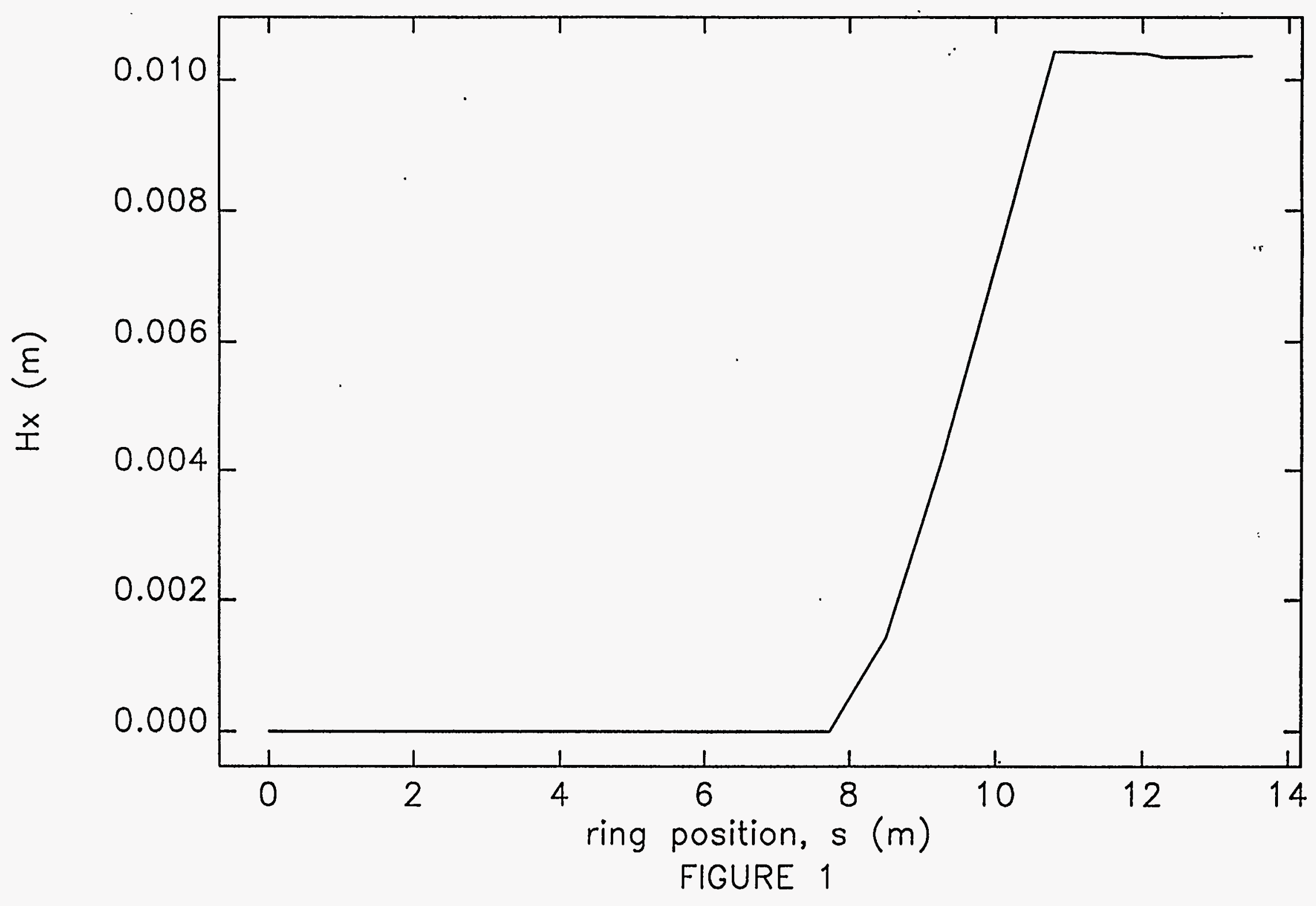



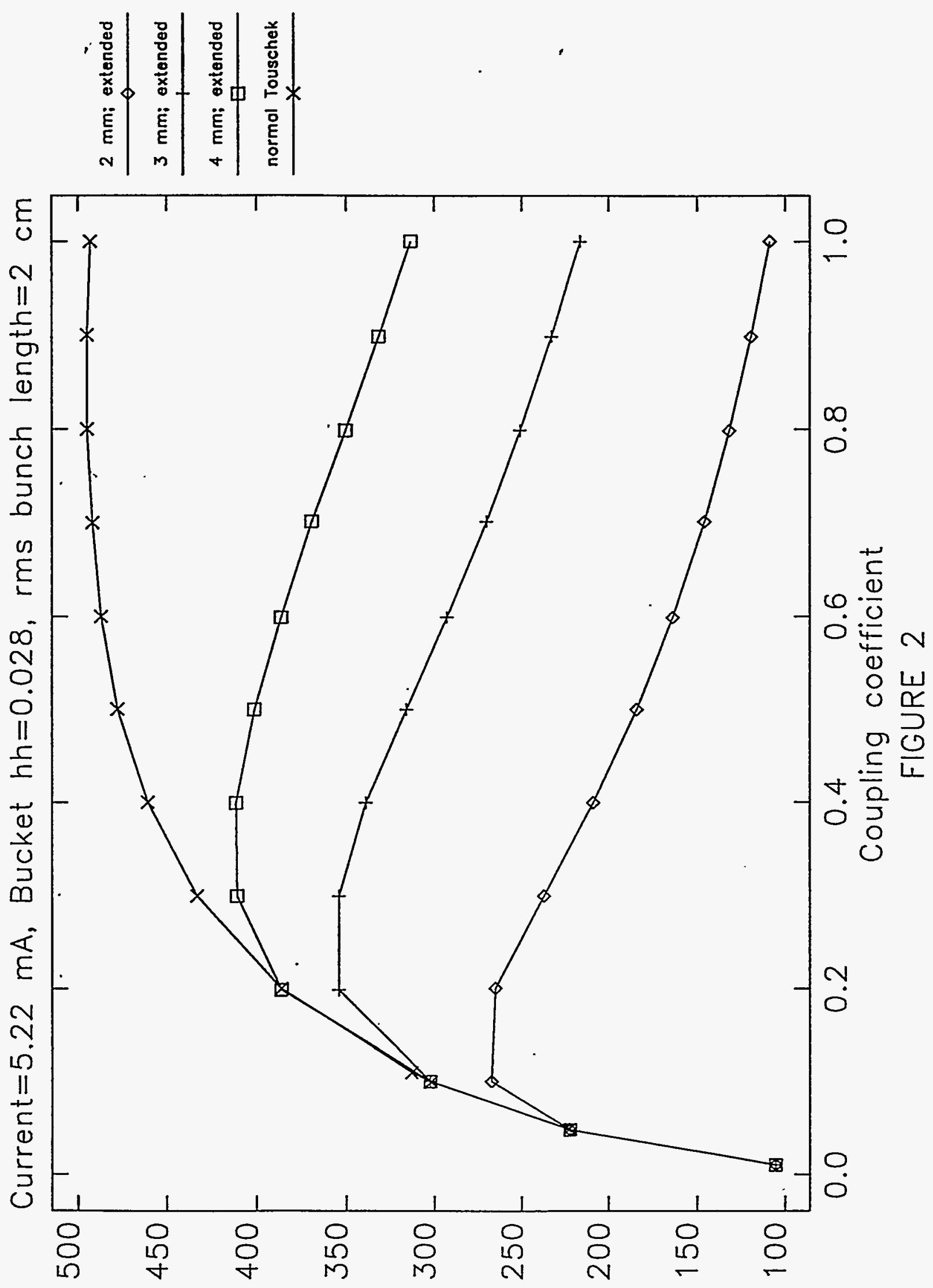

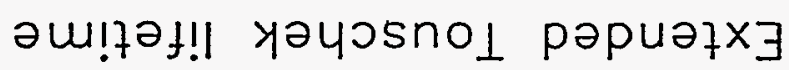




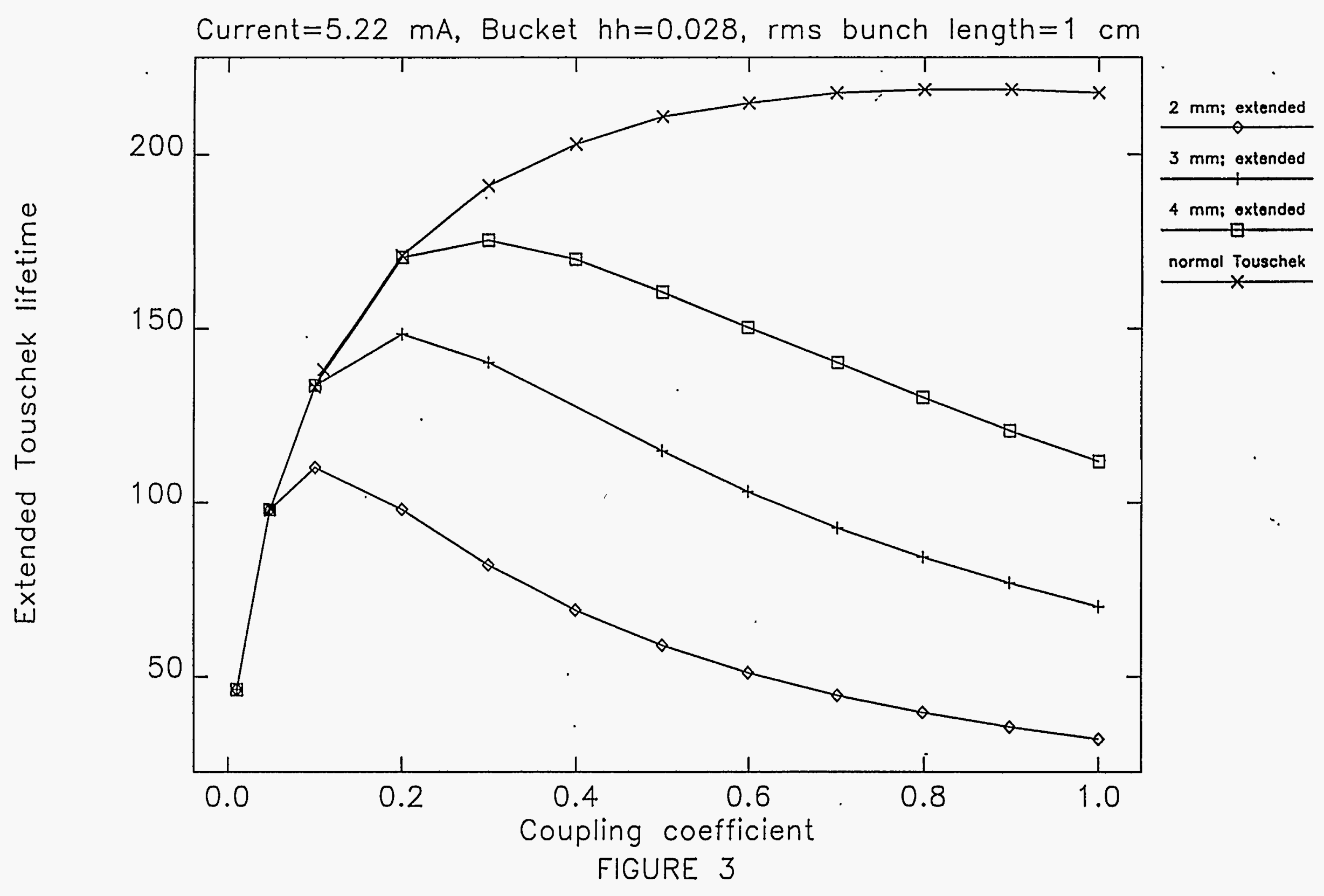




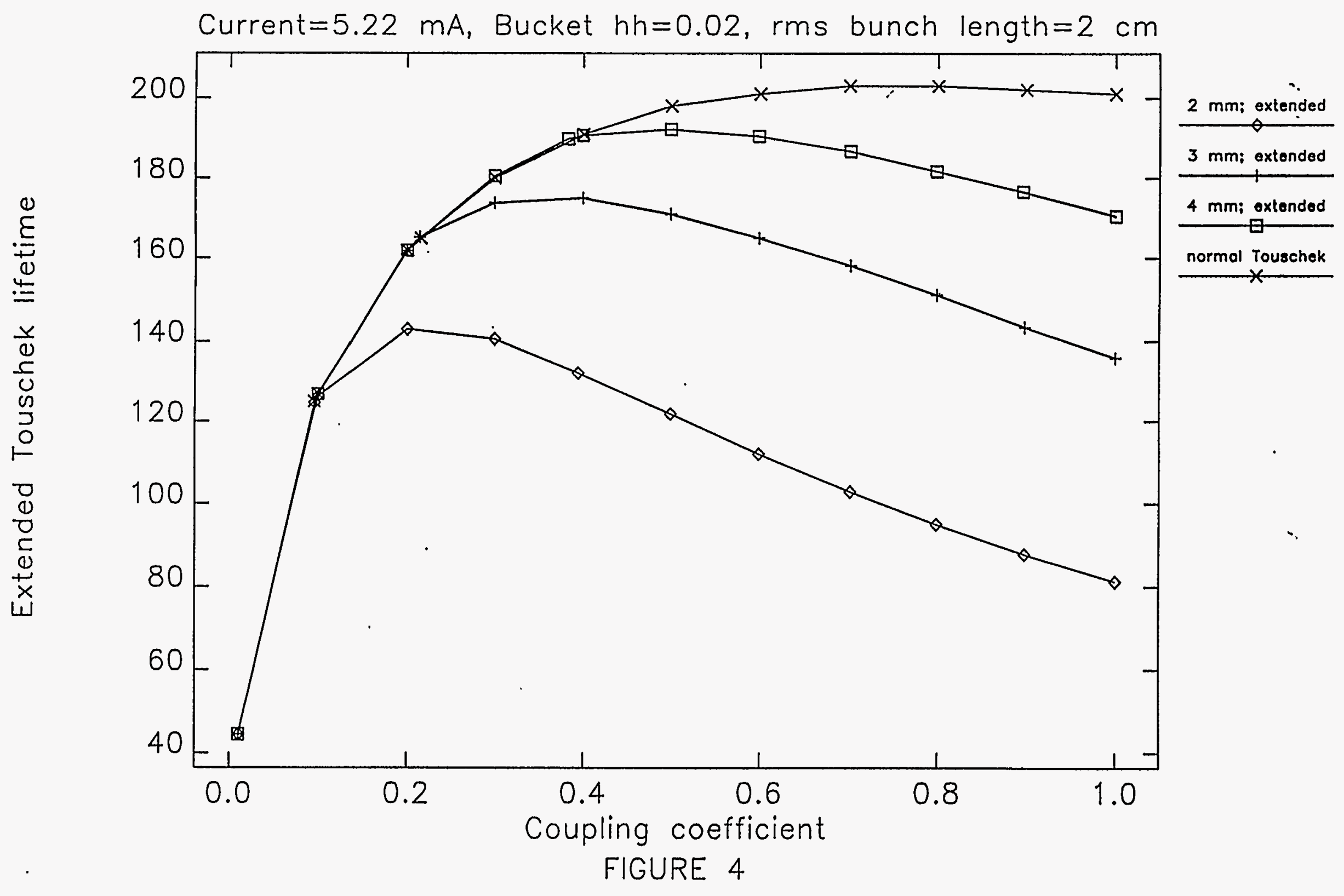




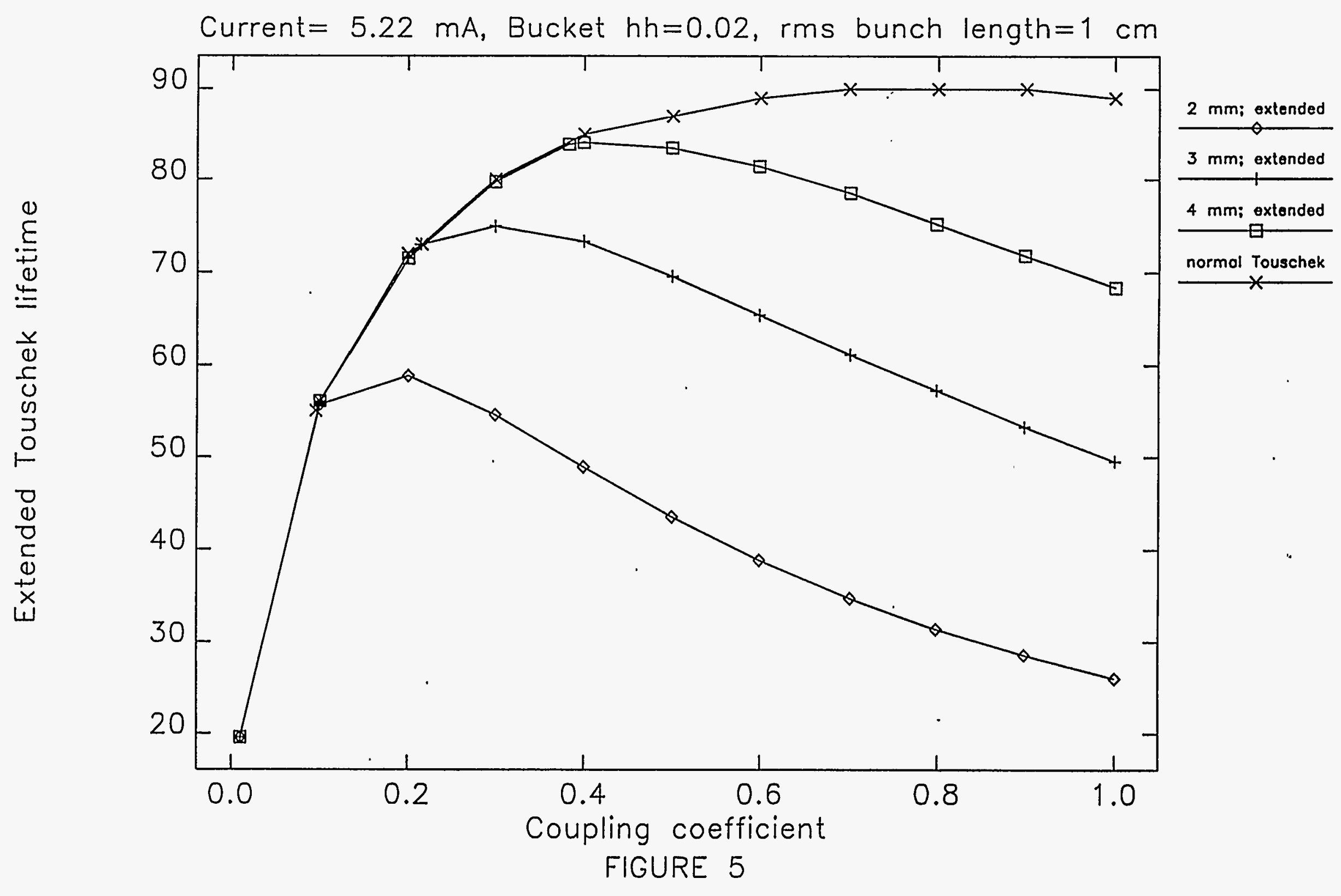




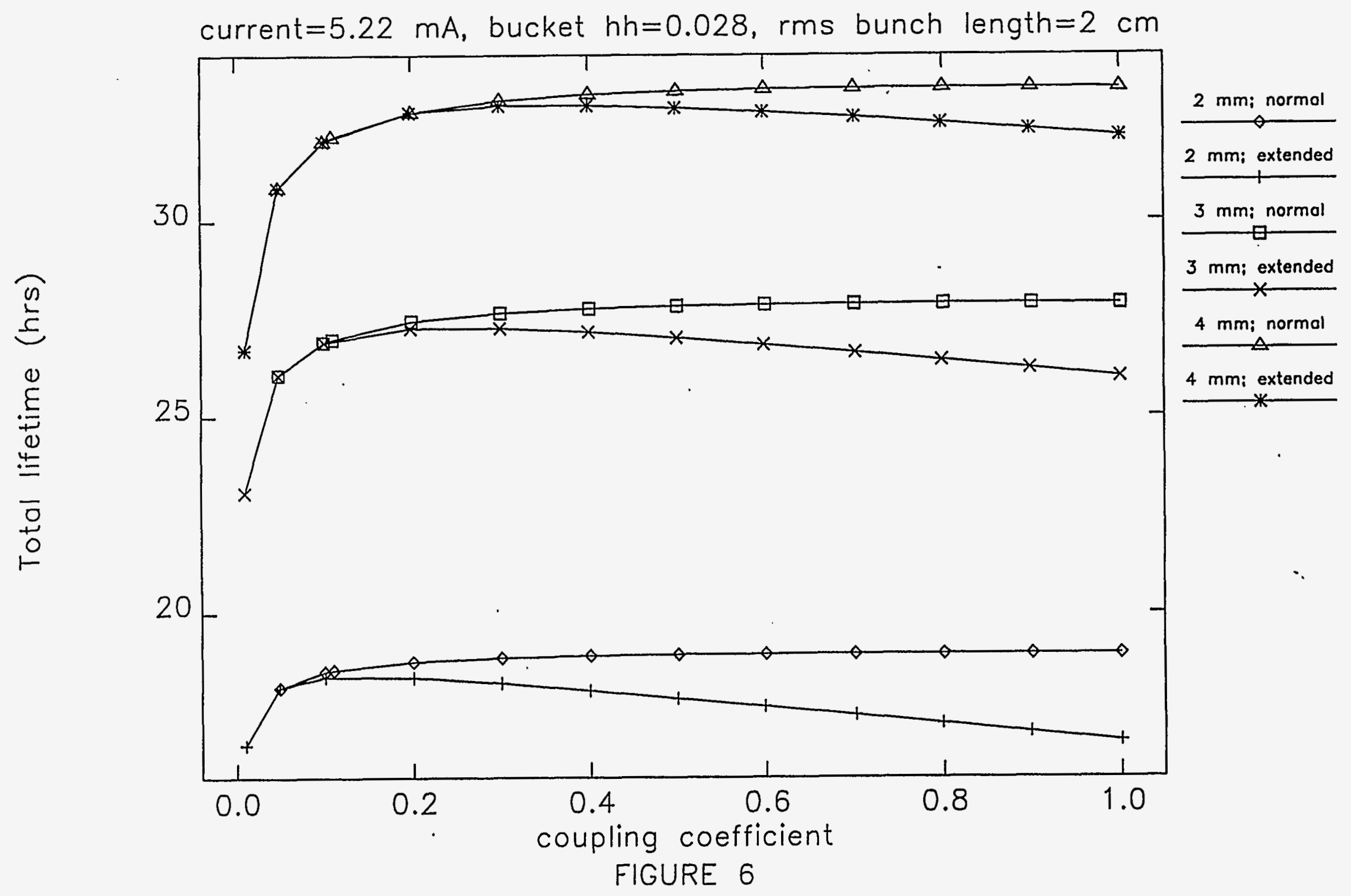




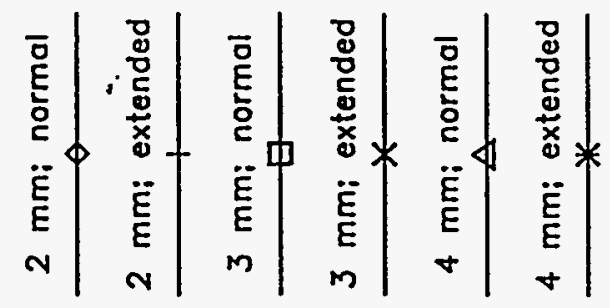

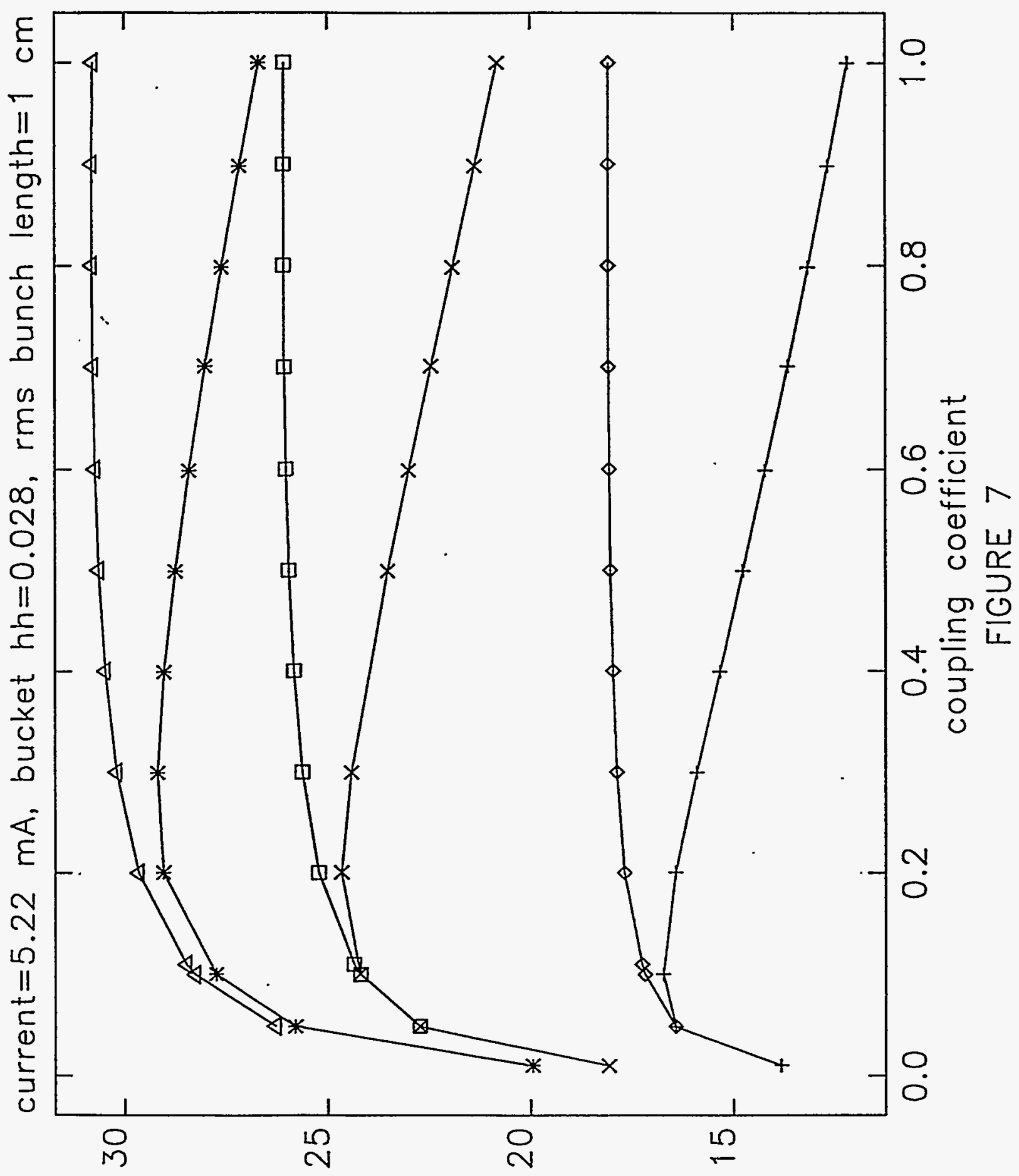

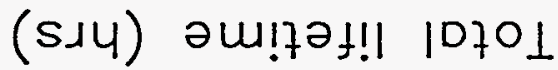




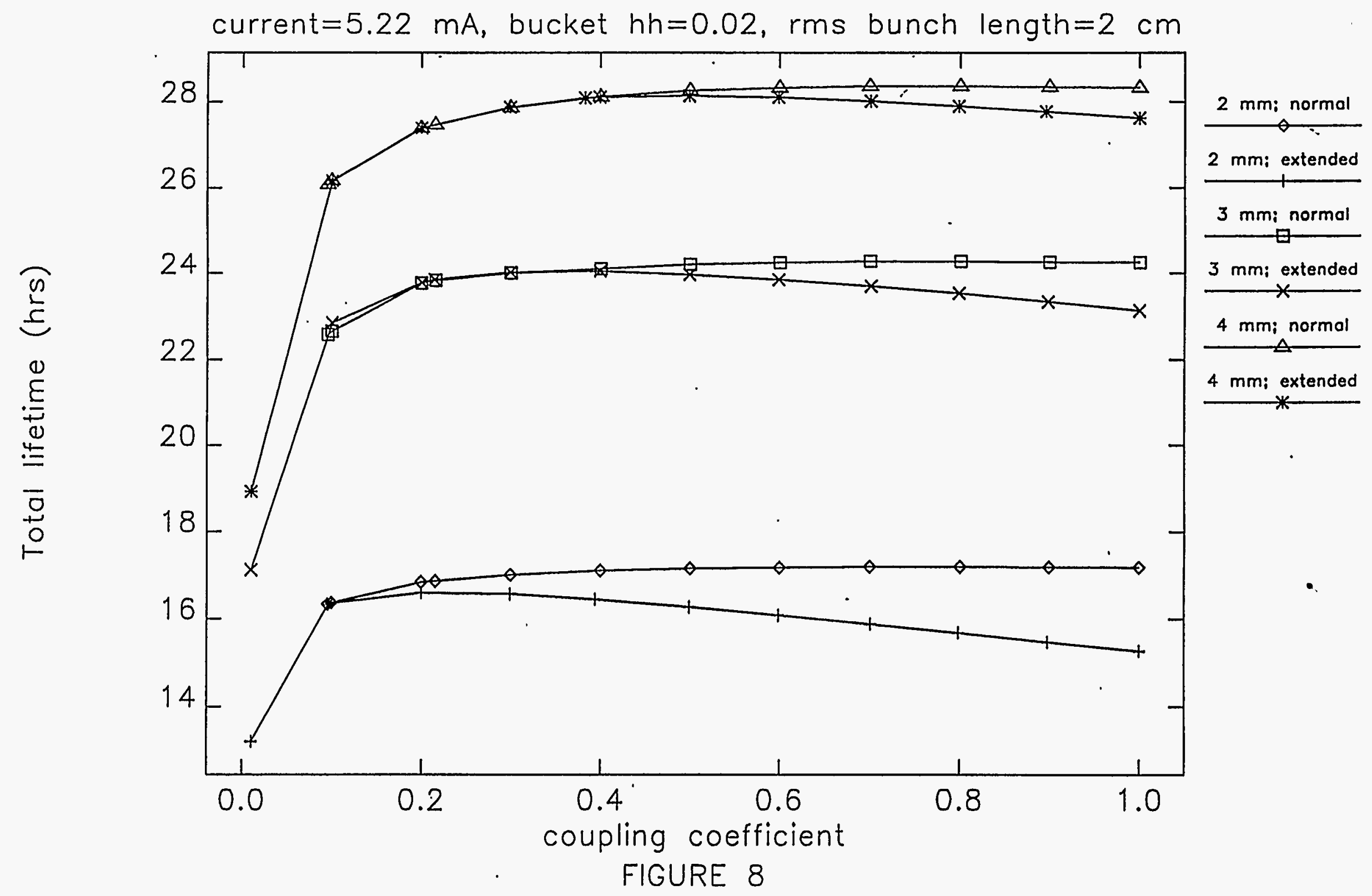




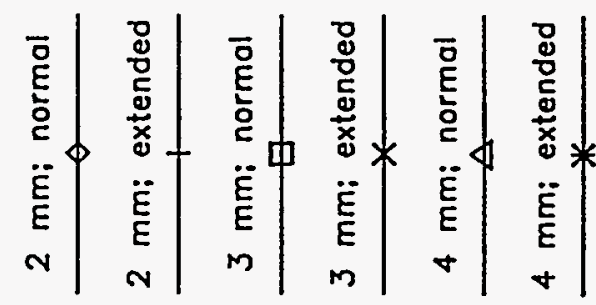

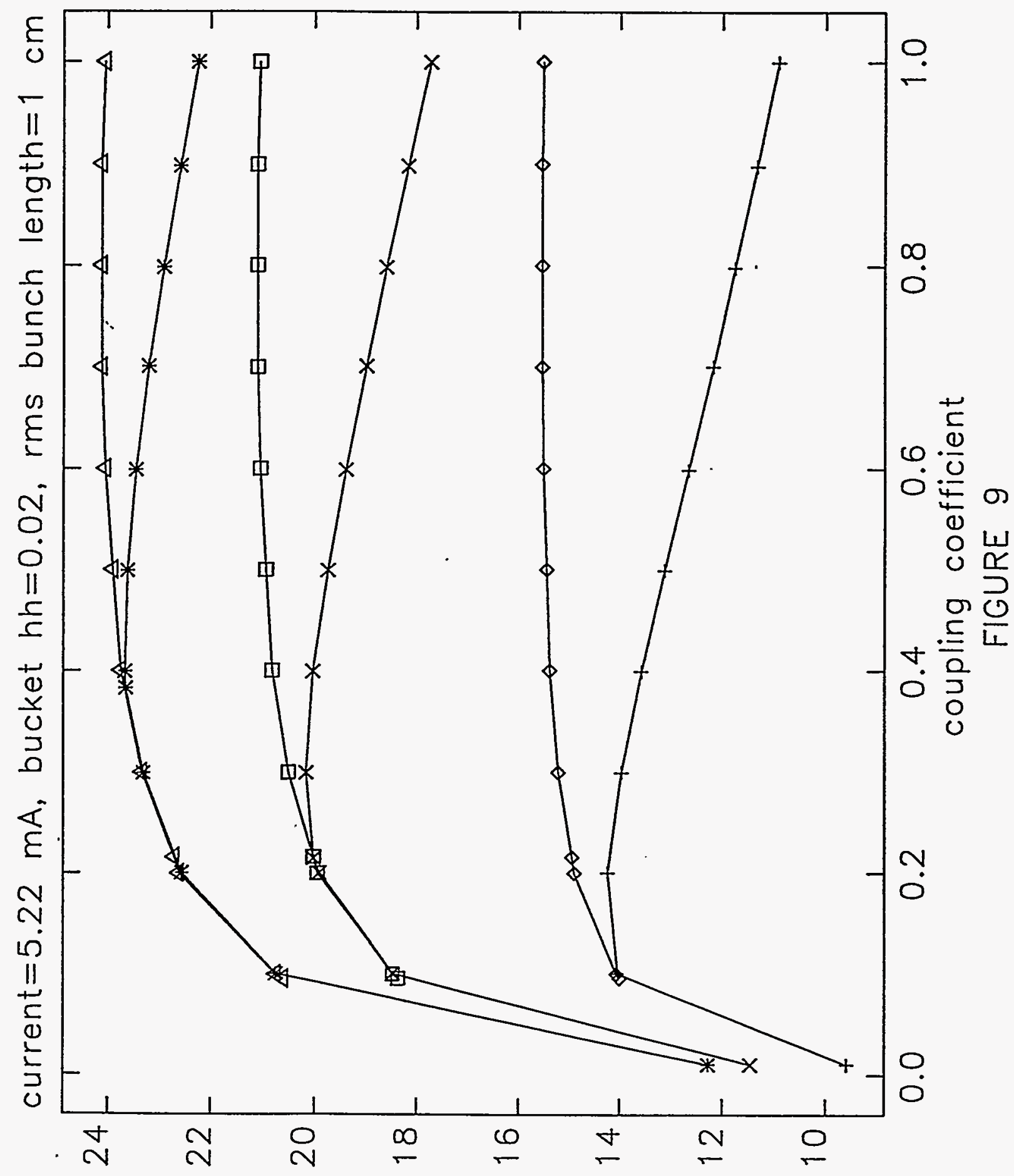

(sג4) әu!7ә!!! 10701 


\section{DISCLAIMER}

This report was prepared as an account of work sponsored by an agency of the United States Government. Neither the United States Government nor any agency thereof, nor any of their employees, makes any warranty, express or implied, or assumes any legal liability or responsibility for the accuracy, completeness, or usefulness of any information, apparatus, product, or process disclosed, or represents that its use would not infringe privately owned rights. Reference herein to any specific commercial product, process, or service by trade name, trademark, manufacturer, or otherwise does not necessarily constitute or imply its endorsement, recommendation, or favoring by the United States Government or any agency thereof. The views and opinions of authors expressed herein do not necessarily state or reflect those of the United States Government or any agency thereof. 